\title{
Invigorating Cancer Prevention: Proposing Core Competencies in Cancer Prevention Research Training
}

\author{
Jessica Faupel-Badger • Carrie Cameron • Shine Chang
}

Published online: 20 July 2013

(C) The Author(s) 2013. This article is published with open access at Springerlink.com

Successes in cancer prevention research over the past 60 years have altered clinical care and had broad implications for public health. Notable examples include the development of chemopreventive agents (e.g., tamoxifen reducing breast cancer risk), vaccines against infectious agents known to cause cancer (hepatitis B and human papillomavirus), screening and early detection targeting groups at higher risk (colonoscopy for ages 50 and older), and recommendations for lifestyle modifications, some of which have also been accompanied by policy efforts (smoking cessation programs and bans on smoking in public places).[1,2] Yet despite these laudable advances, there is a pervasive sense among both the public and those in the field that "we should be further along in preventing cancer." This rigorous science and decades of work required for discoveries to be translated into public health practice seem to be largely underrecognized by both the public and scientific community. When advances in cancer research are highlighted, prevention of cancer rarely takes center stage. Even in this time when emphasis is placed on finding higher quality, lower cost health interventions in general, the word 'prevention'

Jessica Faupel-Badger and Carrie Cameron contributed equally to the development and writing of this article and should be acknowledged as co-first authors.

J. Faupel-Badger

National Cancer Institute, Bethesda, MD, USA

C. Cameron $\cdot$ S. Chang $(\bowtie)$

Department of Epidemiology, The University of Texas MD

Anderson Cancer Center, Mail Code 1340, P. O. Box 301439,

Houston, TX 77230-1439, USA

e-mail: shinechang@mdanderson.org may not even be mentioned. ${ }^{1}$ This state of affairs has led us to contemplate whether a new view of cancer prevention research training is necessary to energize this field and accelerate progress in preventing cancer.

This new proposal for cancer prevention research training would embrace a competency-based model. Employing a competency-based model in cancer prevention training would enable trainees to develop a wider and more effective skill set than that obtained from traditional disciplinespecific research training. The goal is to prepare trainees to work across the diversity of disciplines involved in this field, responding to - and capitalizing on - the dynamic and collaborative nature of the research enterprise and to contribute with high impact to the field's mandate to improve public health. Competency-based training has been explored in many areas, such as medicine, clinical research, global health, public health, biomedical basic sciences research, and these existing models can be informative to the design of specific competencies for cancer prevention research training.

Before considering a similar shift to specific competencies for cancer prevention research training, however, it is important to articulate a vision of what qualities the ideal cancer prevention researcher should embody in order to design objectives and competencies congruent with this vision. As discussed by Frenk and colleagues with regard to the medical curriculum example [4], traditional curricula are often artifacts of historical legacy, designed with little intention-in Frenk's words, "[n]ot uncommonly schools change the objectives to meet what the faculty want to teach so that the curriculum drives the objectives, rather than the

\footnotetext{
${ }^{1}$ See, e.g., the Viewpoint in the April 4, 2013 issue of the Journal of the American Medical Association, an important position statement entitled, "The Future of Biomedical Research," [3] which contains no mention of prevention.
} 
wished-for learning objectives driving the curriculum." Traditional research training curricula are presumably no less faculty-driven than medical training, and prepare students to compete in a system where rewards and promotion are based on intensive single-discipline specialization and publication productivity rather than on the solution of complex problems across disciplines and translation phases.

To meet the challenges of organizing collaboration across disciplines and along the continuum from discovery to application, cancer prevention scientists need leadership and advocacy skills, in addition to broad scientific knowledge and the ability to communicate across disciplines. Leaders also must understand the social and political context of their work to bring public attention and stimulate a sense of urgency in funding agencies. These types of professional competencies have not typically been included in research training, which has focused on acquisition of in-depth, specialized knowledge of a discipline, mastery of particular research methodologies, maturation of the ability to formulate research questions, and development of scientific communication skills-for an audience of similar scientific specialists. Producing researchers with proposed skills in leadership, advocacy, and communication will require a "transformative" approach to education [4-6], which aims to go beyond incremental acquisition of knowledge to bring about a developmental shift towards a more engaged, leadership-driven professionalism.

To identify specific competencies for cancer prevention research, we can benefit from competency models developed for other areas of postgraduate research training. The National Postdoctoral Association (NPA) has identified six core competencies for postdoctoral fellows in research training positions, including both discipline-specific and broader professional development skills [7] (NPA Core Competencies Toolkit). Frameworks for research competencies have been created for medical residents required to conduct scholarly activities through research rotations. These have been organized around specific research tasks (e.g., critiquing the literature, understanding epidemiological and biostatistical concepts applied to diagnostic tests, designing research protocols [8]) or based on specific ACGME-defined competencies (i.e., medical knowledge, interpersonal and communication skills, and professionalism [9]). Other initiatives proposed for competencies in cancer care may be useful for determining content for cancer prevention competencies [10, 11]. An especially instructive example for transforming health professional training comes from the shift in medical residency training over the past decade from testing of acquired knowledge [4] to demonstrating competence in six core areas: patient care, medical knowledge, practice-based learning and improvement, interpersonal and communication skills, professionalism, and systems-based practice. The process of completing this shift is well documented in the literature, from identifying the needs and creating competencies, to designing evidence- based interventions, to developing rigorous evaluation tools. This potent example from medical education suggests that the field of cancer prevention can derive much benefit if we apply such strategies to preparing the next generation of scientists in cancer prevention.

The examples of existing competency models provided here overlap in several areas, yet there are important gaps when we examine their application to cancer prevention research. For example, the NPA competencies for leadership and management skills, while appropriate for the research postdoctoral level, are broadly stated and do not address field-specific challenges or leadership issues within a large, collaborative enterprise. Another example of a competency that is critical to research in many disciplines but may be inadequately specific for successful cancer prevention research is in communication and collaboration. The breadth of cancer prevention as a field and its goal to translate research discoveries into clinical and population practice both demand expertise in communication and collaboration skills and make their mastery difficult. That said, because seeking mastery of such skills for transdisciplinary collaboration may not be an intuitive behavior [12], nor one widely rewarded by academia, which historically has rewarded individuals, not teams, structured training programs that help individuals develop such skills play a critical role. Their professional preparation will be needed as evidence builds for team collaboration as a necessary strategy and potent tool for solving the more intractable or complex research questions previously unassailable by more conventional single-discipline approaches [13, 14].

Here, we have focused on the need to develop competencies for the postgraduate cancer prevention research workforce with the aim of equipping individuals with the credibility and necessary skills to be leaders advancing the field. Cancer prevention, by its nature, requires a multidisciplinary team-based approach, and communicating progress can be challenging. Furthermore, the stakes are high when moving research to practice. Interventions, which aim to prevent cancer in a currently healthy population, require intense examination of the strength of the evidence, biases, risks, and benefits. The ultimate indicator of success in this field is a decline in cancer incidence at the population level, which takes years to achieve and stems from multiple sources. For these reasons, the field's new vanguard needs a broad understanding of the many different stakeholders in this community and how to work effectively across these areas. Creating a system of widely recognized training competencies for cancer prevention research would alleviate some of the heavy expectations upon those entering this field and would clearly articulate outcomes of this training. This model of professional development also would require structured training programs and other approaches designed for teaching team science skills and strategies in order to prepare researchers for focused but collaborative efforts to unravel and intervene upon the complex diseases that afflict 
populations in the twenty-first century. We invite others to join us in thinking critically about the important competencies for cancer prevention and infrastructure to support this type of training needed for not only those engaged in postdoctoral research, but for all constituencies of the cancer prevention workforce, and we welcome your comments and suggestions. We will continue to deliberate on specific recommendations and will put these forth in a subsequent communication.

Open Access This article is distributed under the terms of the Creative Commons Attribution License which permits any use, distribution, and reproduction in any medium, provided the original author(s) and the source are credited.

\section{References}

1. Lippman SM, Hawk ET (2009) Cancer prevention: from 1727 to milestones of the past 100 years. Cancer Res 69(13):5269-5284

2. Umar A, Dunn BK, Greenwald P (2012) Future directions in cancer prevention. Nat Rev Cancer 12(12):835-848

3. Emanuel E (2013) The future of biomedical research. JAMA 309(15):1589-1590

4. Frenk J et al (2011) Health professionals for a new century: transforming education to strengthen health systems in an interdependent world. Lancet 376:1923-1958
5. Mezirow J (2000) Learning as transformation: critical perspectives on a theory in progress. Jossey-Bass, San Francisco

6. Stevens-Long J, Schapiro S, McClintock C (2012) Passionate scholars: transformative learning in doctoral education. Adult Educ Q 62(2):180-198

7. National Postdoctoral Association (2009) NPA Postdoctoral Core Competencies Toolkit, 2007-09. http://www.nationalpostdoc.org/ competencies. Accessed 6 June 2013

8. Kanna, B., et al. 2006. The research rotation: competency-based structured and novel approach to research training of internal medicine residents. BMC Medical Education, 6. DOI: 10.1186/14726920-6-52.

9. Adkison L, Glaros A (2012) Assessing research competency in a medical school environment. Med Sci Educ 22(3S):139-142

10. C-Change (2013) Sustaining a strong national cancer workforce. http://c-changetogether.org/workforce. Accessed 4 April 2013.

11. Smith AP et al (2009) A competency-based approach to expanding the cancer care workforce: proof of concept. Medsurg Nurs Off J Acad Med-Surg Nurs 18(1):38-49

12. Colditz GA, Wolin KY, Gehlert S (2012) Applying what we know to accelerate cancer prevention. Sci Transl Med 4(127):127rv4 127rv4. doi:10.1126/scitranslmed.3003218

13. Hall KL et al (2012) Assessing the value of team science: a study comparing center- and investigator-initiated grants. Am J Prev Med 42(2):57-163

14. Lichtveld $M$ et al (2012) Creating a sustainable cancer workforce: focus on disparities and cultural competence. In: Georgakilas A (ed) Cancer prevention - from mechanism to translational benefits.. doi: $10.5772 / 2253$ 